\title{
Encefalopatía asociada a Valproato
}

\author{
Pablo Young, Bárbara C. Finn, Florencia Álvarez, Julio E. \\ Bruetman, Hernán Trimarchi.
}

\section{Valproate-associated \\ hyperammonemic encephalopathy. Report of one case}

Valproate can be associated to hyperammonemic encephalopathy, characterized by fluctuating sudden-onset alterations of sensorium, focal symptoms and an increase in the frequency of seizures. We report a 78 year-old female using valproate 1,000 mg/ day for 10 months for the treatment to tonic-clonic seizures. She was admitted on three occasions in the last fourth months for self limited clouding of sensorium. Laboratory, imaging and electroencephalografic studies were non-contributory. Blood ammonia levels were $123 \mu \mathrm{g} / \mathrm{dl}$ (normal: 15-50 $\mu \mathrm{g} / \mathrm{dl}$ ). Due to the possibility of a hyperammonemic encephalopathy secondary to valproate, the drug was discontinued and she was treated with lactulose and intravenous $\mathrm{L}$ carnitine, $1 \mathrm{~g} /$ day. The patient showed a complete recovery within 48 hours. This drug-associated encephalopathy is a reversible but potentially fatal cause, probably underdiagnosed, that requires a high index of suspicion (Rev Méd Chile 2007; 135: 1446-9).

(Key words: Carnitine; Encephalopathy; Hyperammonemia; Valproic acid)

Recibido el 28 de septiembre, 2006. Aceptado el 5 de marzo, 2007.

Servicios de Clínica Médica y Nefrología, Hospital Británico de Buenos Aires, Argentina.

$\mathrm{E}^{\prime}$ valproato es un anticonvulsivante efectivo en epilepsias generalizadas o parciales ${ }^{1,2}$. Aunque usualmente es bien tolerado, se ha asociado a varios efectos colaterales sistémicos y neurológi$\cos ^{2}$. Estos últimos incluyen vómitos, mareos, ataxia, trastornos de la marcha y diplopía, si bien los más frecuentes son el temblor y la hipersomnolencia, siendo esta última una probable traducción de una discreta encefalopatía ${ }^{2}$. Ocurre durante los primeros días de tratamiento, correla-

Correspondencia a: Dr. Pablo Young, Hospital Británico. Perdriel 74 (1280) Buenos Aires, Argentina. Tel: 541143096400 Fax: 5411 43043393. E mail: pabloyoung2003@yahoo.com.ar cionándose con los niveles séricos y revierte con la reducción o suspensión de la medicación ${ }^{2}$.

Como efecto adverso infrecuente y grave del valproato se ha descrito la elevación de enzimas hepáticas e hiperamonemia por insuficiencia hepática. Esto se observa luego de comenzado el tratamiento y es más común con la combinación de drogas anticonvulsivantes ${ }^{3,4}$.

Presentamos el caso de una paciente con diagnóstico de encefalopatía hiperamonémica inducida por valproato (EHIV).

\section{Caso Clínico}

Mujer de 78 años con antecedentes de hipertensión arterial, fibrilación auricular crónica, gamapatía mo- 
noclonal de significado incierto, en tratamiento por 10 meses con valproato $1 \mathrm{~g}$ /día por convulsiones tónico clónicas. La paciente presentó tres hospitalizaciones en cuatro meses por episodios breves de deterioro del sensorio con reversión espontánea. En la última hospitalización presentó parámetros vitales normales y un Glasgow 11/15 (respuesta verbal confusa y apertura ocular al estímulo doloroso, localiza al dolor), nistagmus a la mirada lateral.

El laboratorio evidenció hemograma, coagulograma, urea, creatininemia, sodio, potasio, magnesio, calcio, fósforo, hepatograma, glucosa, hormonas tiroideas, folatos, vitamina B12, anticuerpos antiperoxidasa, factor antinuclear, látex, anti Ro, anti La, VDRL y análisis de orina normales. La punción lumbar, el examen toxicológico, hemocultivos, urocultivo y ácido orótico en orina fueron negativos. Una reevaluación de su gamapatía con punción de médula ósea fue normal. La TAC de cerebro mostró imagen secuelar occipital izquierda y la radiografía de tórax fue normal. El EEG evidenció actividad continua lenta generalizada compatible con encefalopatía metabólica. Dosajes de valproato: $67 \mu \mathrm{g} / \mathrm{ml}$ (normal: 50-100) y de amonio: $123 \mu \mathrm{g} / \mathrm{dl}$.

Se interpretó el cuadro como EHIV suspendiéndose el valproato y administrándose lactulosa $10 \mathrm{ml} \mathrm{c} / 8 \mathrm{~h}$ y L-carnitina $1 \mathrm{~g}$ iv/día; se rotó el anticonvulsivante a lamotrigina. Hubo recuperación completa a las $48 \mathrm{~h}$ de la suspensión de la medicación con normalización de la amonemia. A cinco meses la paciente no repitió más episodios.

\section{DisCUSIÓN}

La hiperamonemia es una emergencia médica e inmediatamente se deben tomar los recaudos para minimizar el daño cerebral permanente ${ }^{3-5}$.

Weng sugiere que el amonio debe ser medido en todo paciente con delirio o estupor de causa no aclarada ${ }^{5}$. Es un estudio sencillo y disponible en la mayoría de los hospitales. Aunque la causa más común de hiperamonemia es la alteración severa de la función hepática, también ha sido observada en su ausencia (Tabla 1). En la encefalopatía hepática severa la incidencia de hiperamonemia trepa al $90 \%{ }^{6}$. Otras causas son la infección urinaria por gérmenes productores de ureasa (Proteus mirabilis, Corynebacterium urealyticum), drogas (valproato, 5-fluorouracilo), malignidad y desórde- nes del ciclo de la urea ${ }^{3-5,7}$. En pacientes con mieloma múltiple y deterioro del sensorio la hiperamonemia debe considerarse junto a la hipercalcemia, a la hiperviscocidad y a infecciones ${ }^{8}$.

La excreción de nitrógeno es principalmente realizada en forma de urea (80\%) por riñón ${ }^{5,9}$.

La EHIV es más frecuente en pacientes con deficiencia de carnitina o alteraciones enzimáticas congénitas del ciclo de la urea ${ }^{3,4,10}$. Nuestra paciente presenta un hepatograma normal y ácido orótico negativo en orina lo que hace improbable un desorden del ciclo de la urea.

En pacientes que reciben valproato el amonio puede estar elevado en $16 \%$ a $52 \%$ de los casos sin presentar síntomas neurológicos, y no siempre los síntomas se correlacionan con los niveles de $\operatorname{amonio}^{3,4,11}$.

La ingesta moderada de proteínas puede causar aumentos en la amonemia en pacientes con valproato, y estos cambios no se relacionan con los niveles séricos de la droga y pueden ser atenuados con L-carnitina ${ }^{12}$. El valproato puede provocar una deficiencia secundaria de carnitina, observándose una disminución de la amonemia con la administración de carnitina ${ }^{3,4,12}$. Esta facilita el transporte dentro de la mitocondria de ácidos grasos de cadena larga para la beta-oxidación; el valproato es un ácido graso de cadena corta que es metabolizado en parte por la beta-oxidación y probablemente interfiera con la acción de la carnitina ${ }^{13}$.

La hiperamonemia sería la causal de los síntomas, ya que los altos niveles de amonio cerebrales alteran el metabolismo neuronal al inhibir la recaptación neuronal de glutamato y la homeostasis de fluidos, ya que la glutamina acumulada aumenta la osmolaridad y el edema astrocitario $^{3,14,15}$. La hiperamonemia puede deberse a un aumento de la producción renal por una reducción de la síntesis de glutamina, a una disminución en la producción hepática de urea por el valproato o por disminución de la disponibilidad hepática de carnitina ${ }^{6,9,14}$.

Los signos típicos son alteraciones fluctuantes de la conciencia, signos focales e incremento de los cuadros comiciales. Estas manifestaciones pueden progresar a ataxia, estupor y $\mathrm{coma}^{3-5}$. Los cambios de conducta pueden simular un estado postictal, problemas psiquiátricos o un estatus epiléptico no convulsivo y se tiende a incrementar la dosis de valproato. La hiperamo- 
Tabla 1. Causas de encefalopatía hiperamonémica del adulto

\begin{tabular}{|c|}
\hline $\begin{array}{l}\text { Congénitas } \\
\text { Desórdenes del ciclo de la urea: } \\
\text { Deficiencia de ornitina carbamiltransferasa } \\
\text { Deficiencia de arginosuccinato sintetasa (Citrulinemia tipo II) } \\
\text { Déficit de carnitina: } \\
\text { Defectos congénitos asociados con fallo hepático } \\
\text { Enfermedad de Wilson } \\
\text { Deficiencia de alfa 1-antitripsina } \\
\text { Adquiridas } \quad \text { Cnfermedad hepática: (causa más común) } \\
\text { Cirrosis } \\
\text { Hepatitis aguda y crónica } \\
\text { Inducida por drogas } \\
\text { Síndrome de Reye } \\
\text { Drogas: } \\
\text { Ácido valproico } \\
\text { 5-fluorouracilo } \\
\text { Asparaginasa } \\
\text { Primidona } \\
\text { Tratamiento con Vitamina D en insuficiencia renal crónica } \\
\text { Malignidad: } \\
\text { Leucemias agudas } \\
\text { Mieloma múltiple (14 casos) } \\
\text { Misceláneas: } \\
\text { Infección urinaria por gérmenes desdobladores de urea } \\
\text { Hidronefrosis } \\
\text { Vejiga neurogénica } \\
\text { Doble sistema ureteropielocalicial } \\
\text { Fístula colo-vesical } \\
\text { Postoperatorio de ureterosigmoidostomia } \\
\text { Postoperatorio de resección transuretral de próstata (2 casos) } \\
\text { Altas dosis de quimioterapia } \\
\text { Postrasplante de médula ósea } \\
\text { Malignidad hematológica } \\
\text { Sobrecrecimiento bacteriano } \\
\text { Síndrome de asa ciega } \\
\text { Empiema por anaerobios (bacteroides fragilis) } \\
\text { Nutrición parenteral crónica (con aminoácidos esenciales) } \\
\text { Deficiencia adquirida de carnitina } \\
\text { Hemodiálisis crónica } \\
\text { Tratamiento con ácido valproico } \\
\text { Cirrosis hepática }\end{array}$ \\
\hline
\end{tabular}

nemia crónica produce atrofia cerebral, degeneración quística, agrandamiento ventricular, y retraso en la mielinización ${ }^{16}$. El fenobarbital, la fenitoína, la carbamacepina y el topiramato pueden exacerbar la EHIV.
Ante la sospecha de EHIV se debe discontinuar el valproato y evaluar la función hepática? ${ }^{9}$ El cuadro en general es de buen pronóstico si se diagnostica tempranamente. El diagnóstico diferencial de EHIV se debe realizar con otras causas 
de encefalopatía, tales como las metabólicas, eléctricas, estructurales, tóxicas, farmacológicas e infecciosas y menos frecuentes el estupor migrañoso, la obstrucción intermitente del líquido cefalorraquídeo, la narcolepsia, y el estupor por endozepinas ${ }^{17}$.

El tratamiento incluye discontinuar la droga, hidratación endovenosa, minimizar la produc-

\section{REFERENCIAS}

1. Duncan JS, Sander JW, Sisodiya SM, Walter MC. Adult epilepsy. Lancet 2006; 367: 1087-1100.

2. Johannessen CU, Johannessen SI. Valproate: past, present, and future. CNS Drug Rev 2003; 9: 199216. (Review).

3. Verrotti A, Trotta D, Morgese G, Chiarelli F. Valproate-induced hyperammonemic encephalopathy. Metab Brain Dis 2002; 17: 367-73. (Review).

4. Segura-Bruna N, Rodriguez-Campello A, Puente V, Roquer J. Valproate-induced hyperammonemic encephalopathy. Acta Neurol Scand 2006; 114: $1-7$.

5. Weng TI, Shin FFY, Chen WJ. Unusual causes of hyperammonemia in the emergency department. Am J Emerg Med 2004; 22: 105-7.

6. Brusilow SW. Hyperammonemic encephalopathy. Medicine (Baltimore) 2002; 8: 240-9. (Review).

7. De Jonghe B, Janier V, Abderrahim N, Hillion D, Lacherade JC, Outin H. Urinary tract infection and coma. Lancet 2002; 360: 996.

8. Kwan L, Wang C, LevitT L. Hyperammonemic encephalopathy in multiple myeloma. N Engl J Med 2002; 346: 1674-5.

9. Mathias RS, Kostiner D, Packman S. Hyperammonemia in urea cycle disorders: role of the nephro- ción endógena de amonio, el catabolismo proteico, la ingesta de nitrógeno y su absorción con lactulosa, administrar sustratos para las enzimas del ciclo de la urea (arginina y citrulina endovenosa), administrar sustratos que faciliten la remoción del amonio por vías alternativas (fenilacetato y benzoato de sodio), L-carnitina y eventualmente diálisis.

logist. Am J Kidney Dis 2001; 37: 1069-80. (Review).

10. Coulter DL, Allen RJ. Secondary hyperammonemia. A possible mechanism for valproate encephalopathy. Lancet 1980; 1: 1310-1 (Letter).

11. Murphy JV. Valproate-induced hyperammonemic encephalopathy. Epilepsia 2003; 44: 268; author reply 268.

12. Gidal Be, Inglese CM, Meyer JF, Pitterle Le, Antonopolous J, Rust RS. Diet and valproateinduced transient hyperammonemia: Effect of Lcarnitine. Pediatr Neurol 1997; 16: 301-5.

13. Galan Ortega A, Padros Fluvia am, Hernández Perez JM. [Carnitine deficiency: clinical significance]. Med Clin (Barc) 1998; 110: 426-30. (Review).

14. Bachmann C. Mechanisms of hyperammonemia. Clin Chem Lab Med 2002; 40: 653-62. (Review).

15. Benarroch EE. Neuron-Astrocyte Interactions: Partnership for Normal Function and Disease in the Central Nervous System. Mayo Clin Proc 2005; 80: 1326-38.

16. Blindauer Ka, Harrington G, Morris GL, Ho KC. Fulminant progression of demyelinating disease after valproate-induced encephalopathy. Neurology 1998; 51: 292-5.

17. Cortelli P, Avallone R, Baraldi M, Zeneroli ML, Mandrioli J, Corsi L, ET AL. Endozepines in recurrent stupor. Sleep Medicine Reviews 2005; 9: 477-87. 